Extrême-Orient Extrême-Occident

\section{Extrême-Orient Extrême-Occident}

38 | 2014

La Guerre en perspective : histoire et culture militaire en Chine

\title{
Le stratège comme maître des signes : art de la guerre et art sémiotique en Chine ancienne
}

The Strategist as Master of Signs: the Art of War and the Art of Semiotics in Early China

作為符號大師的戰略家：古代中國的戰術和符號藝術

\section{Albert Galvany}

\section{(2) OpenEdition}

Journals

Édition électronique

URL : http://journals.openedition.org/extremeorient/373

DOI : 10.4000/extremeorient.373

ISSN : 2108-7105

Éditeur

Presses universitaires de Vincennes

Édition imprimée

Date de publication : 1 novembre 2014

Pagination : 65-98

ISBN : 978-2-84292-414-0

ISSN : 0754-5010

Référence électronique

Albert Galvany, « Le stratège comme maître des signes : art de la guerre et art sémiotique en Chine ancienne », Extrême-Orient Extrême-Occident [En ligne], 38 | 2014, mis en ligne le 01 janvier 2017. consulté le 19 avril 2019. URL : http://journals.openedition.org/extremeorient/373 ; DOI : 10.4000/ extremeorient.373 


\title{
Le stratège comme maître des signes : art de la guerre et art sémiotique en Chine ancienne
}

\author{
Albert Galvany ${ }^{1}$
}

[...] tout ce que vous lisez, je suppose, dans le récit d'un narrateur militaire, les plus petits faits, les plus petits événements, ne sont que les signes d'une idée qu'il faut dégager et qui souvent en recouvre d'autres, comme dans un palimpseste. De sorte que vous avez un ensemble aussi intellectuel que n'importe quelle science ou n'importe quel art, et qui est satisfaisant pour l'esprit.

Marcel Proust, Le Côté de Guermantes.

Sous l'influence tenace d'une tradition qui reste vivante et qui persiste à rattacher les débats d'idées de la Chine pré-impériale à des lignées doctrinales bien délimitées, la littérature militaire a été traitée, à de rares exceptions près, comme un corpus textuel unitaire et isolé du reste des lignes de pensée de cette période. Il suffit néanmoins de jeter un regard dépourvu d'à-priori sur l'ensemble de la production textuelle de la Chine ancienne pour se rendre compte à quel point la description de ce paysage intellectuel, fragmenté en aires parfaitement définies et étanches, n'est que le résultat d'une projection sur l'antiquité des catégories ultérieures et souvent étrangères aux textes. En effet, sous la dynastie des Han, l'ensemble des textes anciens qui nous ont été transmis ont été divisés et classés en écoles qui, pour la plupart, ne correspondent pas à des notions dont on pourrait certifier la validité historique au sein de cette littérature même et que l'on pourrait partant assumer sans difficulté, mais

1. Je tiens à remercier Jean Levi, Romain Graziani et Anne-Hélène Suárez qui ont relu, corrigé et commenté le manuscrit de cet article. Leurs remarques pertinentes et judicieuses auront permis d'améliorer sa qualité. 
qui obéissent plutôt à des besoins de catalogage bibliographique ${ }^{2}$. C'est le cas de l' «école militaire» (bing jia), qui offre en l'occurrence un exemple paradigmatique. Le souci de la guerre et des questions militaires, même dans leur dimension la plus technique et spécialisée, est non seulement présent dans les textes traditionnellement assignés à cette école, mais semble également, sans doute en raison du rôle central de ces affaires pendant la période des Royaumes Combattants, déborder de cette étiquette pour se répandre dans une grande partie des écrits philosophiques et politiques de l'époque. Aussi, et afin d'atteindre une meilleure compréhension de ces documents, convient-il, à mon avis, d'aborder leur lecture et leur analyse d'un point de vue plus large, moins soumis au schéma trop rigide qui découle de cette organisation héritée. En accord avec la volonté qui anime ce numéro spécial consacré à la guerre, mon article cherche à briser quelques unes des idées reçues sur la façon dont on a étudié jusqu'à présent la littérature militaire dans la Chine ancienne, en offrant pour cela une perspective alternative.

Loin, donc, de concentrer mon regard exclusivement sur les écrits catalogués ultérieurement dans la catégorie «école militaire», cet article tente d'aborder la figure du stratège telle qu'elle qu'on la trouve décrite dans d'autres sources textuelles de la Chine ancienne à vocation politique, philosophique ou historique, comme un individu capable d'anticiper les événements, de dévoiler la condition réelle de l'autre, de prévoir les plans et les intentions de l'adversaire. D'après une grande partie de la littérature ancienne, le succès de toute action, et surtout des manœuvres militaires, dépend du degré d'adéquation des acteurs à la conjoncture spatiale et temporelle ainsi que de leur capacité à prévoir les situations et d'agir en conséquence. Ce sont justement ces qualités prédictives qui définissent bien souvent le sage et le distinguent du sot; comme nous le verrons dans les pages qui suivent, les façons de concevoir la figure du stratège convergent sur ce point. En effet, les attributs caractéristiques du sage dans une grande partie de la littérature politique, philosophique, voire médicale de la Chine ancienne recoupent ceux du bon stratège. Ceci est particulièrement évident en ce qui concerne l'anticipation des événements et le dévoilement de la réalité interne des êtres grâce à la perception attentive des éléments les plus ténus et leur correcte interprétation. De ce point de vue, le général à la tête des armées peut être considéré comme un maître des signes et, par conséquent, l'art de la guerre peut être représenté comme un savoir qui exige, entre autres vertus, la connaissance de techniques «sémiotiques » qui garantiraient la juste prévision des événements. Ainsi, je vais en premier lieu offrir une description

2. À ce propos, le lecteur intéressé consultera avec profit les travaux de Nylan \& Csikszentmihalyi (2003) et Smith (2003). 
du contexte politique et social, dominé par la nécessité de découvrir l'autre et de déceler sa réalité la plus cachée, dans lequel s'inscrivent ces conceptions, qu'elles soient du sage ou du stratège. J'analyserai plus loin la façon dont les qualités qui découlent de l'anticipation et de l'observation minutieuse des signes interviennent dans une grande partie de la littérature ancienne, en soulignant particulièrement la convergence des procédés et des opérations mentales, des techniques divinatoires à l'inférence à partir d'éléments révélateurs, au sein des récits militaires. Pour finir, et cela dans le but de vérifier les hypothèses que j'ai avancées, je me pencherai sur la biographie de Sun Bin, célèbre stratège de l'antiquité, telle que la retracent les Mémoires Historiques (Shiji) de Sima Qian car, à mon avis, elle fournit l'approche emblématique, ou si l'on préfère, le récit paradigmatique des bénéfices et des risques liés à l'anticipation par le biais d'indices et de traces qui peuvent s'avérer aussi bien vrais que faux. À travers les faits et les gestes de Sun Bin rapportés par Sima Qian, on peut voir se déployer presque sous la forme d'une épure les principaux traits des formes particulières de la pratique de la guerre et de la façon de la penser à l'époque des Royaumes Combattants.

\section{L'univers sur une carapace de tortue}

La popularité des procédés physiognomoniques (xiang) et morphoscopiques (xing $f a$ ) dans la Chine pré-impériale ne fait aucun doute malgré l'absence de tout traité complet et du caractère fragmentaire des manuscrits récemment mis au jour par l'archéologie qui rend leur lecture et leur interprétation matériellement difficiles. L'ampleur de leur diffusion et de leur impact se manifeste non seulement dans la littérature ancienne qui rapporte de nombreux épisodes d'interventions et de cas traités par les spécialistes de ces techniques, mais aussi à travers les critiques et condamnations que certains penseurs importants de l'époque se voient contraints de formuler par écrit dans le but de neutraliser leur pouvoir et leur influence croissantes ${ }^{3}$. On peut comprendre que les gouvernants d'alors, confrontés à l'obligation de sélectionner des

3. À cet égard, le chapitre «Contre la physiognomonie» (Fei xiang 非相) du Xunzi 荀子 et le chapitre «Le seigneur He» (He shi 和氏) du Han Feizi 韓非子 constituent, sans aucun doute, deux exemples révélateurs de l'autorité et du prestige dont jouissent alors ces experts. L'influence et la popularité de la physiognomonie vont s'accroître sous la dynastie Han et plusieurs auteurs importants de cette période vont écrire sur ce sujet, entre autres, Wang Chong 王充 et Wang Fu 王符. Le lecteur intéressé peut consulter, parmi d'autres, le livre par Zhu Pingyi (1990). 
conseillers et des fonctionnaires pour leur administration, éprouvent le besoin d'avoir recours à un certain nombre de procédés. Partant de l'observation du physique, de l'expression, de la posture, de la voix ou d'autres traits extérieurs, ces techniques assurent pouvoir révéler l'identité intime des candidats, aussi bien leur capacité de travail que leur loyauté et leurs qualités morales. S'il est vrai que de telles investigations portent fréquemment sur les êtres humains (du nouveau-né mâle d'un gouvernant au candidat à quelque fonction officielle), l'utilisation de méthodes visant à établir une corrélation entre l'organisation externe d'un objet et ses qualités internes ne se limite pas à ceux-ci. De fait, les spécialistes de ces techniques n'hésitent pas à les étendre à d'autres catégories considérées comme d'intérêt par les élites politiques et sociales du moment: chevaux $^{4}$, pierres présumées précieuses ${ }^{5}$, chiens $^{6}$, épées ${ }^{7}$ voire bois des arbres ${ }^{8}$

4. Les anecdotes dont Bo Le 伯樂, légendaire physiognomoniste équin, est le protagoniste sont recueillies dans de nombreux textes de la Chine pré-impériale (Xunzi, Zhuangzi, Han Feizi, Lüshi chunqiu, Huainanzi, etc.) et rendent compte de la pertinence de tels procédés. Outre les témoignages contenus dans les textes transmis, on a exhumé en 1973 sur le site archéologique de Mawangdui 馬王堆 un manuscrit sur soie intitulé «Le classique de la physiognomonie équine» (Xiang majing 相馬經). Concernant ce document, je me réfère à Li Ling (1993): 1-10.

5. Voir l'anecdote concernant le seigneur He insérée dans le Han Feizi: Han Feizi xin jiao $z h u$ 韓非子新校注, XIII, 271.

6. Dans la tombe numéro 1 du site archéologique de Shuanggudui 雙古堆, on a découvert un manuscrit fragmentaire composé de vingt lamelles de bambou dédiées à la physiognomonie canine (xiang gou 相狗). Pour une information et une description concernant ces matériaux, voir Hu Pingsheng (1998). Par ailleurs, sur le site archéologique de Yinqueshan 銀雀山 ont été récupérés quelques fragments sur ce même sujet en 1972, intitulés par les éditeurs «Recettes de physiognomonie canine» (Xiang gou fang 相狗方). Il est fort probable que parmi ces écrits concernant les techniques physiognomoniques aient pu figurer d'autres textes dédiés à l'examen des animaux domestiques que l'on n'a pas conservés, dans la mesure où l'Histoire des Han (Hanshu 漢書) mentionne un ouvrage perdu composé de six rouleaux (juan 卷) intitulé «La physiognomonie des six animaux domestiques» (Xiang liu chu 相六畜), indiqué dans la partie de la bibliographie correspondant à la «Morphoscopie» (xingfa 形法): Hanshu 漢書, XXX, 1775.

7. Sur le site archéologique de Juyan 居延 on a récupéré plusieurs manuscrits concernant la physiognomonie des épées regroupés sous le titre de «Physiognomonie des épées et dagues précieuses» (Xiang baojian dao 相寶劍刀). Pour une étude de ces matériaux, voir, entre autres, les articles de Ma Mingda (1982) et de Zhong Shaoyi (1994).

8. Dans le Zhuangzi 莊子, il est fait état d'une anecdote dont le protagoniste est un maître charpentier accompagné d'un disciple et dont l'argument central concerne la manière de repérer le bon bois grâce à la reconnaissance des caractéristiques extérieures de l'arbre. Voir Zhuangzi jishi 莊子集釋, IV, 170-171. 
constituent autant d'éléments susceptibles d'un examen mené selon de telles techniques d'observation. Le regard physiognomonique présuppose un monde saturé de signes, débordant de symptômes. À l'intérieur d'un tel univers lisible, un état des choses déterminé, accessible empiriquement, fait référence à un autre qui échappe à la perception ordinaire.

Cette propension à examiner minutieusement la réalité intérieure des objets et des personnes ne se limite pas au recours à de tels procédés divinatoires. En réalité, il s'agit d'un souci fort répandu qui imprègne également d'autres domaines comme, par exemple, et de manière paradigmatique, celui du rituel. Dans le Classique des Rites (Liji), le rite est décrit comme un moyen permettant de déchiffrer les dispositions intérieures des participants aux cérémonies protocolaires, voire de porter un diagnostic sur la solidité des institutions d'un pays. La réalité de telles dispositions qui, en règle générale, restent opaques et inaccessibles au regard des profanes devient visible pour qui connaît les arcanes du rite et sait observer avec pertinence les manières d'être, tout extérieures, des participants ${ }^{9}$. De fait, selon ces doctrines du rituel, la réalité intérieure, voire l'avenir d'une personne peuvent être déchiffrés à partir de ses gestes et de ses expressions, de la manière, par exemple, dont elle marche et déplace ses pieds. L'anecdote suivante tirée des Discours des Royaumes (Guoyu) est, à cet égard, éclairante: au cours de la réunion politique d'ambassadeurs de plusieurs royaumes dans la localité de Keling - située dans la partie occidentale du pays de Zheng - le duc Xiang Dan, conseiller du souverain de Chu, rencontre le prince Li de Jin. Celui-ci affiche alors un regard hautain et se déplace en levant les pieds de manière inconsidérée. Après avoir noté de tels signes certes discrets, mais significatifs, le duc Xiang Dan s'entretient avec le prince de Lu et lui annonce les ennuis à venir pour le royaume de Jin. Surpris par une telle prédiction, le prince de Lu lui demande ce qui l'a conduit à ce pronostic. Le duc de Xiang Dan en est convaincu: il suffit d'observer attentivement le mouvement des pieds du prince et l'orientation de son regard pour en inférer le malheur qui menace son pays ${ }^{10}$. Le pas précipité et le regard trop lointain du prince de Jin révèlent un manquement aux règles protocolaires qui, tôt ou tard, provoquera inévitablement une altération des relations sociales. Tout cela, par conséquent, est interprété par le duc Xiang Dan comme un présage de ce qui va nécessairement se produire. Cette anecdote ne constitue pas une exception: dans plusieurs passages de la littérature ancienne, une erreur ou une entorse au rituel sont interprétées comme les symptômes certains d'un

9. Liji jijie 禮記集解, IX.2, 607 et X.2, 662.

10. Сиоуи 國語, 89-91. 
désastre imminent ${ }^{11}$. Évidemment, le dévoilement de la réalité intime de l'autre et même la prédiction de son avenir ne se font pas uniquement à travers l'observation minutieuse des mouvements, des postures et des attitudes. Entre également en jeu l'analyse du ton et des nuances de la voix, ou encore de la musique jouée par la personne en question ${ }^{12}$. De telles anecdotes soulignent la pertinence de l'axiome selon lequel il est possible de mettre en équation une émotion et son expression, un sentiment intime et sa manifestation somatique. C'est encore dans un passage du Gиоуи que l'on trouve explicitée de manière particulièrement éloquente cette correspondance entre l'observation des signes extérieurs et la révélation d'une intention intime, soulignée, dans le cas qui nous occupe, par une métaphore associée à la chéloniomancie, une technique divinatoire très répandue consistant à interpréter les configurations que dessinent les craquelures provoquées par la chaleur d'un feu intense sur l'écaille d'une tortue ${ }^{13}$. Voici l'anecdote:

Au cours de la rencontre à Guo, Gongziwei de Chu ordonna que deux hallebardiers le précédassent lors de son apparition dans la réunion. Gongsun Guisheng [Zijia] du royaume de Cai alla voir Shusun Muzi en compagnie de Hanhu de Zheng [Zipi]. Muzi dit: «Gonzi de Chu s'est présenté en grand apparat, il ne ressemble pas à un grand ministre, il ressemble plutôt au souverain lui-même.» Zipi de Zheng fit le commentaire suivant: «J'éprouve un certain malaise qu'il se soit fait précéder de deux hallebardiers.» Zijia de Cai répondit: «Chu est un grand pays et Gongziwei est son Premier ministre, ne jouit-il donc pas de la prérogative de se faire précéder de deux hallebardiers?» Muzi répondit: «Cela n'est pas bien. Le Fils du Ciel possède sa garde d'honneur pour que se manifeste l'esprit martial; les chanceliers sont accompagnés de deux chars pour être prêts à envoyer des émissaires; les conseillers sont suivis d'un char afin de pouvoir transmettre les ordres. Si maintenant un chancelier se présente comme s'il était un seigneur féodal, c'est parce qu'il a quelque intention secrète. S'il n'en était pas ainsi, crois-tu qu'il oserait s'entourer de l'apparat d'un seigneur féodal pour se réunir avec les chanceliers des différents royaumes féodaux ? Lorsqu'il retournera, sa condition ne sera plus la même. L'habit est l'ornement du cœur. Il en va ainsi de la tortue: lorsqu'on expose son intérieur au feu, les signes apparaissent à la surface. Si Gongzi de Chu ne parvient pas à se transformer en souverain, il est certain qu'il mourra; il ne se réunira pas une

11. Cf.Chunqiu Zuozhuan zhu 春秋左傳注, Ding Gong 定公, année 15, 1600-1601. Sur cette question, voir Zhang Duansui (1987) : 134-136.

12. Voir, par exemple, Liji jijie, IV.2, 292 et Han Feizi xin jiao zhu, XXXVIII, 913. Voir Kalinowski (1999).

13. Je suis ici la terminologie proposée par Vandermeersch (2013): 26. 
nouvelle fois avec les seigneurs féodaux.» De retour [dans son royaume], Gonziwei assassina Jia' ao et prit sa place sur le trône ${ }^{14}$.

\section{Le sage comme chasseur de symptômes}

$\mathrm{Si}$, pour la mentalité dominante dans la littérature de la Chine ancienne, le monde entier est l'objet d'un examen minutieux et constant, cela tient non seulement à la volonté de sonder la réalité extérieure des personnes et des objets pour en dévoiler les qualités intérieures, mais encore à l'ambition de pouvoir anticiper ainsi une action. Dans les traités politiques comme dans les écrits militaires de l'époque, ce qui distingue l'homme sage de l'individu commun ou du sot, c'est sa capacité de prévoir (xian zhi, xian jian), sa faculté de prédire les événements afin d'agir de manière pertinente. Pour le reste, la pratique physiognomonique, qui n'est rien d'autre que l'art de proposer un jugement sur les personnes ainsi que sur les objets à partir de leurs traits somatiques en leur attribuant une qualité particulière, s'inscrit pleinement dans la logique du paradigme indiciaire tel que défini par l'historien italien Carlo Ginzburg ${ }^{15}$. Selon ce modèle épistémologique, la configuration intérieure des individus et des choses ne peut être déchiffrées qu'au moyen de la détection et de l'interprétation de traces, de symptômes et d'indices.

Les deux notions, l'anticipation d'événements et l'élucidation de signes infimes, sont comme on le voit des notions connexes: de fait, la justesse de la prévision d'événements à venir et du destin d'un individu repose sur la perspicacité du regard. L'homme clairvoyant est, avant tout, quelqu'un susceptible de détecter les symptômes les plus ténus, qui passent inaperçus aux yeux de l'homme ordinaire. Un fragment du Livre du Prince Shang (Shangjunshu), dont les chapitres les plus anciens remontent au Iv ${ }^{\mathrm{e}}$ siècle avant notre ère le dit explicitement dans cette formule proverbiale: «Le sot n'aperçoit que les faits avérés, alors que le sage voit ce qui est à peine en germe $^{16}$.» La visualisation de ce qui n'est pas encore achevé (wei meng), de

14. Gиоуи, 195. Une anecdote consignée dans le Zuozhuan souligne également la correspondance entre habit ou ornement ( $f u$ 服) et la qualité de la personne: Chunqiu Zuozhuan zhu, 638.

15. Ginzburg (1989): 139-180.

16. Shang jun shu zhuizhi 商君書錐指, I, 2. Le même proverbe est à nouveau mentionné, à quelques variantes près, dans le Zhanguo ce: Zhanguo ce jian zheng 戰國策戔證, XIX, 1046; et dans le Xinxu: Xinxu jinzhu jinyi 新序今注今譯, 298. Les réflexions introduites sur ce point à propos de l'intelligence prédictive sont redevables à l'excellent travail de Levi (1989): 30-45. 
ce qui est caché (ming ming), de ce qui n'a pas pris encore une forme (wei xing), constitue le trait définitoire et distinctif du sage dans nombre d'écrits politiques et philosophiques de la Chine ancienne ${ }^{17}$. Prévoir les événements implique donc de se rendre attentif aux détails infimes. En effet, pour qui est doué d'une capacité de perception supérieure, ces minuscules objets épars, quasi imperceptibles, contiennent et annoncent le déroulement d'une séquence d'événements à venir qui, le temps passant, une fois atteint un état critique, trouve sa forme définitive ne permettant plus aucune intervention. La participation active et déterminée au flux continu des événements ne peut se déployer qu'à partir de cette perspicacité prévoyante qui fournit au sage une marge de manœuvre confortable, mais qui reste inaccessible à ceux qui se contentent d'en saisir la structure figée. Le Han Feizi offre, à cet égard, une anecdote significative. Intégrée au chapitre dédié à gloser le Laozi, elle vise à illustrer une maxime contenue dans ce texte - «Prévoir le difficile dans le facile, agir sur ce qui est grand à partir de ce qui est petit ${ }^{18} »$, en faisant le récit d'une intervention du célèbre médecin Bian Que:

Bian Que examina le duc Huan de Cai. Au bout d'un moment, il déclara: «Vous êtes atteint d'une maladie qui affecte pour l'instant la surface de la peau, mais si l'on n'intervient pas immédiatement, elle risque de pénétrer plus profondément dans l'organisme.» Le duc répondit: «Je n'ai rien de tel.» Après son départ, le duc fit le commentaire suivant à son entourage: «Ah! ces médecins, ça ne pense qu'à leur propre prestige en soignant des maladies que vous n'avez pas.» Dix jours plus tard, Bian Que fut reçu une nouvelle fois en audience. Et il mit à nouveau le monarque en garde: «Le mal a maintenant traversé la peau et, si l'on n'intervient pas, il risque de pénétrer plus profondément dans l'organisme.» Le duc ne répondit pas, et afficha une mine contrariée après le départ de Bian Que. Dix jours passèrent avant que le médecin n'examinât à nouveau le duc et déclarât: «La maladie a atteint l'estomac et les intestins; si l'on n'intervient pas, elle va encore s'aggraver.» Le duc ne répondit pas et, après son départ, il se montra taciturne. Dix jours plus tard, Bian Que aperçut le duc et décida de passer son chemin. Le duc dépêcha quelqu'un l'interroger à ce propos et voici quelle fut sa réponse: «Quand on détecte un mal à la surface de la peau, des compresses chaudes suffisent; lorsqu'il a pénétré la peau, l'acupuncture fait l'affaire; même lorsque la maladie se manifeste dans l'appareil digestif, une décoction peut se révéler efficace. Mais sitôt qu'elle pénètre dans les

17. Guanzi jiaozhu 管子校注, I, 17; Shuo yuan jiaozheng 說苑校証, XVI, 397-398; Chunqiu fanlu yizheng 春秋繁露義證, IV, 131; Kongcongzi 孔叢子, XXI, 274; Lun heng jiaoshi 論衡校釋, LXXVIII, 1072; Shiji, XLIII, 1807.

18. Boshu Laozi jiao $\mathrm{zhu}$ 帛書老子校注, 133 . 
os, le cas ne relève pas du médecin mais du Directeur du Destin ${ }^{19}$. Or le mal dont souffre le duc a pénétré les os. Quel remède pourrais-je lui proposer désormais?» Cinq jours plus tard, le duc commença à éprouver des douleurs dans tout le corps. Il fit appeler Bian Que, mais ce dernier était déjà parti pour le pays de Qin. Le duc Huan mourut peu après. Voilà pourquoi, pour traiter les maladies, il faut les combattre lorsqu'elles se manifestent à la surface de l'épiderme: il faut s'attaquer au mal lorsqu'il est dans l'œuf. Ainsi, dans les affaires dont dépendent le bonheur et le malheur, il existe un moment où les choses ne sont encore qu'épidermiques. D'où le dicton: le sage anticipe le flux des événements ${ }^{20}$.

Le fait que le récit du Han Feizi, à visée clairement politique, ait pour cadre l'exercice de la clinique n'est certainement pas indifférent à notre propos. En Chine ancienne, la pratique de la médecine requiert une sensibilité et une vigilance extrêmes pour déceler de bonne heure les symptômes annonciateurs de la maladie. Parce que ces derniers sont découverts au stade le plus précoce, le spécialiste est certain de pouvoir intervenir au plus tôt et, par conséquent, de traiter la maladie avec davantage de chance de succès ${ }^{21}$. Il n'est pas surprenant qu'une autre anecdote, dont Bian Que est à nouveau le protagoniste, rapportée dans le He Guanzi dans un contexte également politique, souligne l'importance essentielle de l'anticipation grâce à l'observation des symptômes, au stade où la maladie commence à peine à se manifester et ne présente pas encore sa configuration achevée. Après avoir rendu hommage à l'habileté de son frère aîné, capable de diagnostiquer une maladie avant qu'elle n'ait pris une forme précise (wei you xing), et avoir décrit l'action des meilleurs médecins, le récit rapporte les propos suivants de Bian Que: «Les médecins compétents savent détecter la maladie chez des patients en bonne santé. Ils traitent le mal avant qu'il ne soit nommé et même avant qu'il ne se soit installé. L'intervention est

19. Pour une étude plus détaillée de cette divinité appelée «Directeur du Destin» (si ming 司命) associée à la durée de la vie et à laquelle on sacrifie dans la Chine ancienne, voir Csiskszentmihalyi (2011).

20. Han Feizi xin jiao zhu, XXI, 440-441.

21. À ce sujet, voir, par exemple, le chapitre «Ba zheng shen ming lun 八正神明論》 du Huangdi neijing suwen 黃帝內經素問: Huangdi neijing zhangju suoyin 黃帝內 經章句索引, 81-83. Par ailleurs, l'observation minutieuse des signes ténus constitue le fondement commun à la conception de la médecine ainsi que d'une grande partie des procédures morphoscopiques dans la Chine ancienne. Il en va de même pour la culture grecque classique où la littérature médicale joue un rôle important dans le développement de la physiognomonie et d'autres techniques de pronostic qui comportent une pratique sémiotique. Pour une étude plus complète de cette question, je renvoie aux travaux de Manetti (1993): 37-52 et de Debru (2007). 
parfaite lorsque les patients sont persuadés que les choses en sont arrivées là par elles-mêmes ${ }^{22}$.» D'où l'insistance dans l'épisode de la maladie du duc Huan rapporté dans le Han Feizi sur la nécessité de faire face aux difficultés dès qu'elles commencent à apparaître sur la peau (cou li), la partie la plus superficielle de l'organisme.

\section{Signes précurseurs et science militaire}

Le terme biao, qui désigne la doublure, la superficie ou la forme extérieure d'un corps ou d'un vêtement, joue aussi un rôle important dans ces valences imaginaires de la surface et de la profondeur. A cet égard, l'une des sections des Printemps et Automnes du seigneur Lü (Lüshi chunqiu) porte pour titre «L'observation des signes extérieurs » (guan biao) et s'occupe précisément de la question de la prévision à long terme des événements, trait qui distingue le sage de l'individu du commun, à partir de l'examen minutieux des signes ténus qu'il est possible de repérer à la surface des êtres :

Le cœur des hommes est tellement caché et voilé qu'il est difficile à percevoir, il est tellement profond et abyssal qu'il est difficile à sonder. C'est pour cette raison que le sage s'attache à observer les intentions. Si le sage se distingue, c'est grâce à sa capacité de prévision. Et la prévision consiste nécessairement à examiner avec soin les signes révélateurs à la surface des choses. Sans de tels signes révélateurs extérieurs, ni Yao ni Shun ne seraient parvenus à l'emporter sur les individus du commun en matière de prévision. [...] Celui qui ne parvient pas à maîtriser une telle méthode, attribue tout les phénomènes à l'action des esprits ou du hasard, alors qu'en réalité ils ne dépendent ni des uns ni de l'autre mais relèvent de cette seule façon de procéder ${ }^{23}$.

Il s'agit, une fois de plus, de pénétrer et d'éclairer l'opaque réalité intérieure des hommes. Bien que ces derniers n'aient de cesse de cacher jalousement leurs projets et leurs désirs dans le fond le plus reculé de leur cœur, selon l'auteur de ce passage, le sage possède la faculté de les dévoiler et de les connaître par avance grâce à une méthode consistant à scruter les signes révélateurs (zheng) qui apparaissent à la surface des choses. Comme souvent dans le Lüshi chunqiu, la construction argumentative de ce chapitre comporte des développements

22. He Guanzi hui jiao jizhu 鶴冠子汇校集注, XVI, 338-339. Voir aussi Yantielun jiao $z h u$ 鹽鐵論校注, LIX, 604.

23. Lüshi chunqiu xin jiao shi 呂氏春秋新校釋, XX.8, 1422. 
théoriques généraux annoncés au début de la partie concernée et complétés par une série d'histoires exemplaires qui servent à accréditer les propos initiaux. Le premier incident, donné à titre d'illustration, concerne le vicomte Cheng de Hou: sur le chemin de Jin, il est hébergé par un hôte du Wey et invité à un banquet; lors de son retour de Jin, au lieu de faire étape à nouveau dans le Wey, le vicomte décide de continuer; interrogé sur la raison de cette décision par l'un des compagnons de voyage, il déchiffre la portée de certains événements survenus durant le banquet qui lui font pressentir le danger qui pèse sur ce pays et la ruine qui le guette. Le second incident met en scène le célèbre stratège Wu Qi. En route vers la cour du pays de Wei, Wu Qi, parvenue à la localité de Anmen, fait une halte pour visiter la région de Xihe et inopinément se met à pleurer à chaudes larmes. Sondé par l'un des siens sur son abattement soudain, Wu Qi lui avoue qu'alors qu'il était encore au service du prince du Wei, il aurait pu soumettre le Qin, son plus redoutable rival, à partir de cette région. Mais le prince avait ignoré ses conseils sur ce point. Il prédit donc que toutes ces terres et le pays de Wei tout entier vont être très bientôt conquis par les troupes de Qin $^{24}$. Ainsi, après avoir rapporté ces deux cas où les protagonistes font preuve d'une prodigieuse capacité pour prédire avec certitude les événements à venir à partir d'un examen minutieux d'éléments du présent apparemment infimes et insignifiants aux yeux de leurs compagnons de voyage, cette partie se clôt avec le passage suivant, qui porte sur les procédés physiognomoniques équins :

Jadis, ils furent des experts en physiognomonie équine: Han Fengshi, qui examinait la denture; Ma Chao, qui examinait la tête; Zi Nüli, qui examinait l'œil; Wei Ji, qui examinait le crin; Xu Bi, qui examinait la croupe; Tou Fahe, qui examinait le poitrail et le flanc; Guan Qing, qui examinait le museau et le cou; Chen Pei, qui examinait les pattes et les sabots; Qin Ya, qui examinait l'avant de l'animal et Zang Jun, la partie postérieure. Ces dix individus furent les plus grands experts de leur temps et bien qu'aucun ne portât son regard physiognomonique sur la même partie, ils observaient tous un seul signe révélateur du cheval qui leur permettait de déterminer la qualité de ses articulations, l'agilité de ses extrémités, sa constitution et sa résistance. Cette méthode d'examen peut s'appliquer non seulement aux

24. La même anecdote concernant Wu Qi figure dans d'autres parties du Lüshi chunqiu intitulées de manière significative «Voir à long terme» (Chang jian 長見) et «Surveillance de l'infime» (Shen xiao 慎小), ces deux fragments également dédiés à la capacité des sages à prévoir les événements à partir de l'examen de signes ténus: Lüshi chunqiu xin jiao shi, XI.5, 612 et XXV.6, 1690. 
chevaux, mais également aux personnes et aux nations qui présentent des signes révélateurs ${ }^{25}$.

Si l'on en croit ce chapitre du Lüshi chunqiu, il existe une continuité évidente et naturelle entre, d'un côté, la capacité du vicomte Cheng de Hou et de Wu Qi à anticiper l'avenir d'un individu ou d'une nation à partir de la lecture de quelques indices subtils et, de l'autre l'habileté de ces experts en physiognomonie à révéler la qualité des chevaux grâce à l'observation d'un trait ayant valeur d'indice. Dans les deux cas, le procédé est identique : le succès des prédictions dépend de l'interprétation des signes révélateurs (zheng) ${ }^{26} .11$ s'agit là d'une notion souvent utilisée également dans la littérature politique pour désigner des facteurs ou des symptômes précurseurs qui permettent de pronostiquer l'ascension ou la ruine d'une nation. Bien que le nombre et la nature de ces facteurs puissent varier d'un texte à l'autre, de nombreux écrits anciens postulent l'existence d'une série d'indices dont l'observation sert au diagnostic de l'état réel des institutions civiles et militaires d'un pays et, par extension, de son avenir ${ }^{27}$. Sensible au besoin impérieux de connaître à l'avance l'état réel et les objectifs de l'adversaire, la littérature stratégique ancienne utilise aussi cette notion pour élaborer une partie de son arsenal théorique. Si le fragment du Han Feizi se rapportant à l'habileté de Bian Que à diagnostiquer précocement la maladie se concluait par l'affirmation que le sage anticipe le flux des événements (sheng ren zao cong shi), cette fonction est remplie, dans le domaine militaire, par le commandant. Selon certains traités de stratégie, c'est à lui qu'incombe la responsabilité d'observer attentivement et de prévoir les mouvements de l'adversaire, si bien que, sans son concours, la connaissance anticipée de l'ennemi est impossible (bing wu zhu, ze bu zao zhi di) ${ }^{28}$. Il n'est donc pas surprenant qu'à partir d'un tel schéma de pensée partagé, les Six Carquois (Liu Tao) comportent un chapitre dont le titre peut être traduit soit par «Les facteurs militaires », soit par «Les signes prémonitoires des questions militaires » (bing zheng). Comme d'autres chapitres de ce même ouvrage, cette partie s'ouvre également sur un dialogue entre le roi Wu et le grand duc Wang à propos de la science militaire. La conversation débute de la manière suivante:

25. Lüshi chunqiu xin jiao shi, XX.8, 1423.

26. Voir Brown \& Bergeton (2008): 649-654.

27. À ce sujet, voir, par exemple, Guanzi jiao zhu, XXIII, 468; Xunzi jijie 荀子集解, X, 194; Han Feizi xin jiao zhu, XV, 300; Xinshu jiaozhu 新書校注, X.2, 380.

28. Guanzi jiao zhu, XVII, 317. Parmi les manuscrits exhumés en 1972 à Yinqueshan, dans la section intitulée «Wang Bing 王兵», on trouve une expression très proche: [ ] 無將不蚤知. Yinqueshan Hanmu zhu jian 銀雀山漢墓竹簡, p. 136. 
Le roi Wu demanda au grand duc: «Existe-t-il donc une manière de connaître par avance le degré de la force ou de la faiblesse d'un adversaire avant de le combattre et de prévoir au moyen de signes révélateurs qui sera vainqueur et qui vaincu?» Le grand duc répondit: «Les signes révélateurs de la victoire ou de la défaite se manifestent par des éléments subtils qui peuvent être perçus par avance. C'est pourquoi, sachant que la défaite dépend des hommes, le commandant clairvoyant prête à ces signes la plus grande attention. Il observe avec une grande égalité d'âme les allées et venues, les progressions et les reculs de son adversaire, il le scrute lorsqu'il est en action comme lorsqu'il est au repos et il examine le ton de ses discours et les échanges de paroles dans les rangs des troupes quand les chefs leur transmettent les ordres. ${ }^{29}$.

La question que le roi Wu pose à son conseiller renvoie, une fois de plus, à l'obligation de connaître par avance et de prévoir (xian zhi, yu jian) le potentiel militaire de l'adversaire. La réponse du conseiller ne fait que prolonger et développer une seule et même idée récurrente: l'observation d'un certain nombre de traits et d'éléments visibles chez l'adversaire révèle les modalités de son comportement qui, à leur tour, permettent de tirer des conclusions générales concernant sa nature ou l'état de ses forces. On trouve un raisonnement quasi identique dans un passage du deuxième chapitre du Wuzi, intitulé de manière significative «Évaluer l'adversaire» (liao di), où le marquis $\mathrm{Wu}$ pose la question suivante au stratège militaire: «Je voudrais percevoir la réalité intérieure de l'ennemi au moyen de l'observation de ses manifestations extérieures, connaître les modalités de son comportement en l'examinant attentivement lorsqu'il est au repos et, ainsi, établir avec certitude l'issue du conflit.Puis-je connaître votre opinion à ce sujet ${ }^{30}$ ? » La réponse qu'apporte $\mathrm{Wu} \mathrm{Qi}$, comme les recettes que le grand duc livre à son souverain pour étancher sa curiosité, ont en commun un point fondamental: il convient d'examiner avec la plus grande attention un certain nombre de caractéristiques extérieures de l'adversaire afin d'accéder, par leur intermédiaire, à sa réalité intérieure. Les deux textes fournissent de nombreux exemples illustrant cette

29. Tai gong Liu Tao jinzhu jinyi 太公六鞱今註今譯, XXIX, 135. L'expression yao xiang 祅祥, qui signifie littéralement «mauvais et bons augures», fait ici certainement référence aux rumeurs de type optimiste ou pessimiste circulant parmi les soldats et qui permettent d'évaluer leur degré de cohésion avec les chefs et d'apprécier leur moral. Un autre passage de la littérature militaire, le Wuzi 吳子 en l'occurrence, emploie la même locution pour exprimer une idée identique: Wuzi jinzhu jinyi 吳子今註今譯, II, 83.

30. Wuzi jinzhu jinyi, II, 83 . 
méthode: l'examen minutieux de la manière dont les soldats se mettent en formation ou s'alignent dans certaines circonstances, l'observation des gestes, des regards et des propos que les combattants échangent avant d'entrer en action, des mouvements des drapeaux et des bannières sur le théâtre des opérations, etc., permet d'inférer la discipline régnant parmi les troupes de l'adversaire. En dernière instance, grâce à l'observation et l'interprétation de tels indices, il est possible supposément d'anticiper l'issue de l'affrontement. Dans les Six Carquois (Liu Tao), l'énumération des éléments précurseurs qui servent à faire la lumière sur l'état réel de l'adversaire est suivie de la description d'une méthode d'observation proche, à l'évidence, des procédés divinatoires. Dans l'argumentation développée au chapitre du Lüshi chunqiu dédié à l'observation des traits extérieurs, la capacité de prévision d'événements futurs est décrite à partir de l'examen d'éléments infimes du présent pour en venir ensuite à l'exposé des qualités exceptionnelles de certains experts en physiognomonie équine. Il en va de même avec le chapitre du Liu Tao dédié aux signes prémonitoires. Dans les deux textes, on passe naturellement, sans solution de continuité, de l'explicitation d'une méthode déductive à partir de l'observation de certains indices à la description d'une technique proprement divinatoire. En effet, après avoir détaillé certains traits extérieurs de l'armée adverse qui anticipent son état et son potentiel, le texte poursuit:

En général, lorsqu'on attaque une ville entourée de murailles, il convient de savoir que si une vapeur de couleur grisâtre comme de la cendre éteinte s'échappe de l'enceinte fortifiée, c'est que la cité peut être détruite; si la vapeur qui s'en dégage provient de sa partie Nord, c'est que la ville peut être conquise; si cela se produit dans la partie située à l'Ouest, c'est que sa capitulation est certaine; si elle s'élève dans la partie située au Sud, c'est que la ville ne peut être prise; et si cela se produit dans la partie située à l'Est, c'est que la cité ne doit pas être attaquée. Dans le cas où la vapeur monte pour se dissiper ensuite, cela signifie que le chef de la cité a fui ; si elle enveloppe nos troupes, cela veut dire que ces dernières subiront des pertes. En général, en ce qui concerne l'offensive contre une cité fortifiée, s'il ne tonne ni ne pleut pendant dix jours, il convient d'abandonner le siège car la ville recevra bientôt d'importants renforts. C'est ainsi que l'on peut savoir si une offensive doit être poursuivie ou si, au contraire, il convient de l'arrêter ${ }^{31}$.

Les lignes qui concluent l'intervention du grand duc dans ce chapitre des Six Carquois renvoient, sans doute, à la technique divinatoire connue sous le nom d'«observation des vapeurs» (wang qi), également mentionnée dans

31. Tai gong Liu Tao jinzhu jinyi, XXIX, 136. 
d'autres écrits militaires ${ }^{32}$. Comme nous venons de l'indiquer, l'argumentation exposée dans ce chapitre passe sans heurt d'une inférence tirée de la perception d'indices et de signes ténus à l'exposition d'une méthode divinatoire.

\section{Lire et fabriquer des signes}

Une telle proximité entre ces procédés trouve un précédent significatif dans deux anecdotes quasi consécutives du Zuozhuan qui s'inscrivent également dans un contexte militaire. Dans deux situations différentes, trois personnages s'attachant à un examen scrupuleux de signes ténus et de détails symptomatiques émettent une série de pronostics qui sont le résultat aussi bien d'opérations déductives à partir de l'observation de manifestations que de pratiques ou techniques divinatoires. La première de ces scènes transporte le lecteur au cœur de la bataille de Pingyin, qui oppose les troupes du Qi aux armées du Jin en 554 av. J.-C. :

Le souverain de Qi gravit la montagne Wu pour observer les troupes de Jin. Les hommes de Jin avaient envoyé leur général en chef pour reconnaître les lieux stratégiques dans les hauteurs et dans les basses terres. Ce dernier établit des formations militaires avec des drapeaux y compris dans les endroits que l'armée ne pouvait atteindre. Il envoya des chars de combat avec des soldats à gauche et des mannequins à droite et ordonna qu'ils se déplaçassent en agitant drapeaux et étendards. Le souverain de Qi, voyant tout cela et craignant que les troupes de l'adversaire fussent très nombreuses, ordonna la retraite. Au vingt-neuvième jour [du dixième mois], le dernier jour de la lune, les armées de Qi abandonnèrent leur campement durant la nuit. Shi Kuang dit au monarque de Jin: «Les corbeaux émettent des croassements joyeux; les troupes de Qi ont fui.» Xing Bo dit alors à Zhonghang Bo: «Il y a un bruit lointain de chevaux qui nous parvient; les troupes

32. Un passage du chapitre LVIII du Mozi 墨子 offre une description succincte de cette technique. Grâce à elle, poursuit ce texte, il est possible de déterminer clairement le vainqueur et le vaincu, le fortuné et l'infortuné (Mozi jiangu 墨子間詁, 606). Par ailleurs, au chapitre XV du Huainanzi dédié aux questions militaires, cette technique divinatoire est mentionnée avec d'autres procédures mantiques (Huainanzi jiaoyi, 1558). Il est probable que ces techniques aient été recueillies dans un texte perdu intitulé «Bie Chengzi» 別成子 que cite le Hanshu (Hanshu, XXX, 1760). Pour une étude de ce procédé divinatoire, voir l'article de Hulsewé (1979). 
de Qi ont fui.» Et Shu Xiang dit au monarque de Jin: «Il y a des corbeaux sur les murailles de la ville; les troupes de Qi ont fui.» ${ }^{33}$.

Le général en chef du Jin a su exploiter le caractère timoré du monarque de Qi, lequel, comme indiqué dans cette même partie du texte, manque de bravoure ou de courage ${ }^{34}$. Par un stratagème qui donne à croire qu'il dispose d'une troupe considérable, le stratège de Jin parvient à intimider son adversaire qui, dans la perspective d'avoir à affronter un ennemi nettement supérieur en nombre, décide de fuir le combat. C'est à ce moment-là que le récit introduit le jugement unanime des trois observateurs concernant la retraite définitive des troupes du Qi. Le premier est celui de Shi Kuang, célèbre musicien aveugle et conseiller à la cour du roi Ping de Jin (r. 557-531 av. J.-C.), doté d'une prodigieuse sensibilité musicale et d'un talent politique considérable ${ }^{35}$. Dans l'antiquité, plusieurs sources décrivent ses faits et gestes ainsi que ses talents, tels que prédire les événements à partir de sons et même soumettre les esprits au moyen de la musique ${ }^{36}$. Ici, Shi Kuang conclut que l'ennemi a fui après avoir écouté attentivement les modulations joyeuses du chant de certains oiseaux. Ainsi qu'il a été dit plus haut, selon ce qui est rapporté dans plusieurs sources textuelles de l'époque, les caractéristiques intérieures d'un individu ou le dénouement à venir d'une conjoncture déterminée peuvent être déchiffrés à partir d'une écoute exercée et attentive des sons ${ }^{37}$. Le deuxième observateur, un officier du Jin, parvient à la même conclusion également grâce à l'acuité auditive, dans ce cas la perception du son lointain provenant des chevaux. Bien qu'il semble s'agir ici d'une inférence à partir d'un élément sensible, il n'en reste pas moins certain que l'allusion au bruit des chevaux qui s'éloignent pourrait aussi faire référence à un passage du Zhouyi, dans lequel la glose

33. Chunqiu zuozhuan zhu, Xiang gong, année 18, 1038. Voir aussi Li Wai-yee (2007): 175.

34. Chunqiu zuozhuan zhu, Xiang gong, année 18, 1037.

35. À propos des idées et des activités politiques de Shi Kuang, voir Pines (2002): 139146.

36. Voir, par exemple, Gиоуи, 460-461; Han Feizi xin jiao zhu, X, 205-207; Huainanzi jiaoyi, XI, 1182. Par ailleurs, il faut signaler que le Hanshu comporte le titre d'une œuvre en huit livres ayant un rapport à Shi Kuang ainsi que d'autres ouvrages liés aux techniques divinatoires appliquées au domaine militaire: Hanshu, 1760.

37. Comme ne cesse de l'indiquer une grande partie de la littérature politique et philosophique de la Chine ancienne, la musique et les sons peuvent révéler, tout au moins pour qui est doté d'une oreille sensible et exercée, les qualités intérieures des personnes, des animaux et même des pays. Voir, entre autres références, Kongzi jiayu shu zheng 孔子家語疏証, V, 125. 
de l'hexagramme Zhun («Difficultés initiales») indique à plusieurs reprises: «Chars et chevaux s'éloignent» (cheng maban ru) ${ }^{38}$. Enfin, le troisième observateur, Shu Xian, célèbre officier du Jin, donne pour certaine la fuite de l'ennemi en observant attentivement un signe révélateur: les corbeaux posés sur les murailles de Pingyin, symptôme irréfutable de l'abandon de la ville ${ }^{39}$.

Plus avant dans cette même partie du Zuozhuan, une deuxième scène réitère une grande partie des éléments qui viennent d'être relevés. Il s'agit de l'incursion des troupes de Chu sur le territoire du pays de Zheng, qui se conclut par un échec après le siège infructueux de la capitale et la perte d'une grande partie de ses effectifs en raison du froid et de la pluie lors de leur retraite. Apprenant la nouvelle des manœuvres d'invasion de Chu, trois officiers du Jin se trouvent d'accord pour affirmer que le Chu ne menace pas leurs intérêts:

\begin{abstract}
Après avoir reçu à Jin des nouvelles concernant les manœuvres de Chu, Shi Kuang déclara: «Ils ne pourront pas nous porter préjudice. J'ai souvent interprété les airs du Nord ainsi que les airs du Sud et ces derniers n'ont pas de force, ils sont remplis de sons moribonds. Il est certain que Chu n'aura pas de succès». Dong Shu ajouta: «Le cours céleste se trouve au Nord-Ouest, ce n'est pas un moment propice pour les régiments du Sud, il est certain que [le Chu] ne remportera pas de succès ». Et Shu Xiang s'exclama: «[Le succès] réside dans la vertu du souverain. $»^{40}$.
\end{abstract}

La faiblesse militaire de Chu est prédite à trois reprises par ces différents observateurs, qui recourent à des techniques distinctes. Le premier pronostic porté à nouveau par Shi Kuang, est établi à partir de l'examen comparatif des mélodies et chants caractéristiques du Nord par opposition à ceux du Sud où, précisément, se trouve le Chu. Il se fonde, par conséquent, sur l'application d'une technique divinatoire consignée dans de nombreux textes de l'antiquité consistant en un examen des sons et modulations ${ }^{41}$. La deuxième prédiction

38. Zhouyi zhengyi 周易正義, 135-137.

39. Dans un autre passage du Zuozhuan traitant d'une campagne militaire, les observateurs de Zheng se rendent compte de la retraite des troupes du Chu après avoir aperçu une nuée de corbeaux posée sur le campement ennemi (Chunqiu Zuozhuan zhu, Zhuang gong 莊公, année 28, 242). De la même manière, un fragment de l'Art de la guerre de Sunzi (Sunzibingfa 孫子兵法) qui énumère une série de signes révélateurs servant à préciser l'état réel du combat indique également que «là où se posent les oiseaux, il n'y a pas d'ennemis.» (Shi yi jia zhu Sunzi jiao li 十一家注孫子校理, IX, 198.)

40. Chunqiu Zuozhuan zhu, Xiang gong, année 18, 1043.

41. L'un des cas les plus célèbres concernant la capacité révélatrice de la musique et des sons se trouve dans une anecdote du Zuozhuan à propos de la visite protocolaire du Duc Zha de Wu 公子札 au Duc Xiang de Lu 裹公 en l'an 543 av. J.-C. Le premier émet 
semble relever, bien que de manière non explicite, de l'observation du firmament : il s'agit là d'un procédé divinatoire souvent utilisé dans un contexte militaire fondé sur la correspondance entre les positions des corps célestes et le calendrier $^{42}$. Le troisième diagnostic, enfin, résulte d'une inférence à partir du constat de la vertu du souverain de $\mathrm{Chu}^{43}$. Comme dans le cas de la bataille de Pingyin, les prédictions livrées par ces trois observateurs résultent de procédés différents, mais convergents qui mettent en jeu aussi bien l'observation de symptômes (ou de prodromes) que différentes façon d'établir un diagnostic en continuité avec ces derniers.

La scène de la bataille de Pingyin est un cas exemplaire de cette tendance naturelle à intégrer différents procédés de pronostic dans un contexte militaire. Plus généralement, elle illustre le rôle crucial que l'examen et l'appréciation des signes joue dans l'issue d'un combat dans les écrits politiques et militaires de cette période. Le jugement unanime des trois observateurs à propos de l'abandon du Qi est précédé du stratagème conçu par le commandant en chef de Jin qui tire le meilleur parti de la psychologie de l'ennemi. Cette ruse de guerre consiste à déployer un leurre, dans ce cas les faux soldats, c'est-à-dire un ensemble de signes visuels trompeurs qui seront néanmoins interprétés comme vrais. De ce point de vue, la leçon de cette bataille est évidente: la victoire sur le théâtre des opérations dépend, en grande partie, de la capacité à percevoir correctement les signes extérieurs qui se présentent aux sens et qui permettent d'anticiper les plans et les manœuvres de l'adversaire. Le bon stratège est capable aussi bien de distinguer les fausses pistes et les signes authentiques que de produire chez l'adversaire l'effet inverse en fabriquant des signes destinés à tromper et leurrer.

à cette occasion un jugement sur le climat moral et, par conséquent, sur l'avenir de tous les pays après avoir écouté avec attention leurs différents styles musicaux et leurs compositions: Chunqiu Zuozhan zhu, Xiang gong, année 29, 1161-1166. Concernant le domaine militaire, un passage du Zhouli atteste également le recours à l'analyse des sons en provenance de la bataille pour en pronostiquer l'issue: Zhouli zhengyi 周禮正 義, XLV, 1852.

42. Pour une étude des procédés divinatoires et astronomiques dans un contexte militaire dans le Zuozhuan ainsi que dans d'autres textes de l'antiquité, voir Pankenier (2013): 279-294.

43. Rappelons ici que l'examen ou l'observation de la vertu (de 德) constitue aussi l'un des huit facteurs ou signes révélateurs (zheng 徵) mentionnés dans le Liu tao qui servent à la connaissance de l'état réel de l'adversaire: Tai gong Liu Tao jinzhu jinyi, XX, 112. 


\section{Les signes de l'opprobre et de la renommée}

Pour être efficace, le regard scrutateur et prédictif évoqué précédemment doit faire face à un écueil des plus importants. Contrairement à ce qui se produit lors de l'examen morphoscopique d'objets inanimés comme les pierres ou les épées, voire de certains animaux comme les chiens ou les chevaux, qui constituent des objets passifs, les êtres humains sont dotés de la capacité de feindre et de tromper. Or, comme on l'a vu, la mentalité ritualiste tend à établir une corrélation directe entre intériorité et extériorité. Elle prétend, par conséquent, construire un monde transparent où la qualité morale et l'avenir même des individus sont clairement lisibles à partir des expressions de leur corps. Si bien qu'elle ne parvient absolument pas à dissiper la crainte que certaines de ces actions rituelles puissent ne pas être authentiques. Dans la mesure où les attitudes et les gestes du corps sont imitables et falsifiables, rien ne garantit la sincérité de ces expressions extérieures et, par conséquent, rien ne peut écarter radicalement le soupçon de duplicité. Ainsi la sincérité est souvent la condition nécessaire, mais indémontrable, de cette stricte adéquation entre la forme de l'enveloppe et l'essence cachée dans son fond ${ }^{44}$.

$\mathrm{Si}$, dans un tel contexte rituel, les signes du corps sont susceptibles d'être falsifiés, dans le domaine de la guerre, le recours à la ruse est la norme. De ce fait, les soupçons concernant la véracité des indices révélateurs observables chez l'adversaire se trouvent multipliés. En effet, affranchi de tout engagement moral, le mensonge incarne l'un des piliers centraux de l'activité stratégique. Une célèbre formule de l'Art de la guerre de Sunzi exprime cette idée de manière lapidaire: «La guerre est l'art de tromper ${ }^{45}$.» La simulation et la dissimulation jouent un rôle capital non seulement pour empêcher l'ennemi d'accéder à la situation réelle de son adversaire, mais aussi pour provoquer l'échec de ses plans et manœuvres. Le stratège doit être, par conséquent, un maître de la tromperie: donnant à voir une réalité trompeuse, il se propose d'abuser un ennemi séduit par les apparences pour le faire tomber dans son piège. Le mensonge (gui) auquel se réfère le passage cité du Sunzi renvoie à une constellation de notions proches et récurrentes, comme la ruse $(z h a)$ ou l'artifice (wei), ressortissant à l'aspect irrégulier de l'art de la guerre, à sa dimension d'exception et d'imprévisibilité (qi) qu'un bon général doit savoir utiliser pour remporter la victoire finale. Ainsi, dans la lignée de cette logique de la tromperie et de la falsification, le succès des opérations militaires est

44. Voir Da Dai Lijijiegu 大戴禮記解詁, LXXII, 190; Huainanzi jiaoyi, VI, 632; et Yi Zhoushu hui jiao ji zhu 逸周書彙校集注,LVIII, 774-775.

45. Shi yi jia zhu Sunzi jiao li, I, 12. 
censé reposer, dans une large mesure, sur l'habileté du stratège à fabriquer des leurres et fausses pistes pour intoxiquer l'adversaire.

Le désir impérieux de déchiffrer la réalité des êtres par l'examen minutieux de leur aspect extérieur, le besoin de prévoir les événements dans le but de se projeter sur la chaîne du temps ainsi que cette dialectique entre volonté de savoir et obligation de se cacher en dissimulant ou en falsifiant est le propre de la science militaire. Tous ces éléments se retrouvent de manière particulièrement éloquente dans le récit biographique concernant Sun Bin recueilli dans les Mémoires Historiques ${ }^{46}$. Comme c'est le cas avec d'autres figures illustres évoquées par Sima Qian, le récit ne prétend pas restaurer dans son intégralité la vie et la carrière militaire de Sun Bin (de fait, l'épisode n'apporte que peu d'éléments proprement biographiques: très peu est dit sur sa formation, tous ses faits d'arme n'y figurent pas et le récit ne fournit aucun élément sur la fin de sa vie). Il vise plutôt à condenser quelques-unes des lignes maîtresses de l'œuvre stratégique qui lui est attribuée dans une seule anecdote significative qui, de ce fait, va fonctionner comme une sorte d'emblème ${ }^{47}$. Ainsi qu'on va le voir, l'épisode concernant Sun Bin ne se contente pas d'apporter de nouveaux éléments importants d'un point de vue historique. Il met en jeu toutes les notions stratégiques que nous avons passées en revue : le latent et le manifeste, le visible et l'invisible, l'observation et la prédiction, les bénéfices et les risques de l'anticipation par le biais d'indices qui peuvent se révéler aussi bien vrais que faux. Mais laissons le texte lui-même guider notre démonstration. La notice biographique s'ouvre sur les lignes suivantes :

Cent ans après la mort de Sun Wu, apparut Sun Bin. [Sun] Bin naquit dans les régions de Ai Zhuan [dans le pays de Qi] et il était un descendant direct de Sun $\mathrm{Wu}$. Sun Bin se forma à la science militaire en compagnie de Pang Juan. Pang Juan servait déjà le pays de Wei où il fut nommé général des armées du roi Hui ; conscient du fait que ses capacités ne pouvaient être comparées à celles de Sun Bin, Pang Juan fit convoquer ce dernier en secret. À l'arrivée de Sun Bin, craignant que le talent de son condisciple n'éclipsât le sien et pris d'envie, il manœuvra pour qu'il fût condamné à l'amputation des deux pieds et au tatouage du visage afin de le mettre à l'écart et rendre impossible un entretien [avec le roi].

Le pays de Qi envoya alors un émissaire à [Da] Liang [la capitale du Wei]. Sun Bin, étant mutilé, s'entretint avec lui en secret, mais il sut le persuader. L'émissaire s'aperçut qu'il s'agissait d'un homme exceptionnel et l'emmena à Qi en le cachant

46. Shiji, LXV, 2162-2165.

47. Voir à ce propos, Levi (1995): 155-156; Hardy (1999): 40; et Galvany (2011). 
dans son chariot. Le général de Qi, Tian Ji, avait de la considération pour lui et accepta de le recevoir en tant qu'invité d'honneur ${ }^{48}$.

Bien que succincte, la première partie de la notice biographique de Sun Bin expose avec clarté la manœuvre ourdie contre lui par son ancien camarade d'étude Pang Juan. Conscient du fait que la présence de Sun Bin dans le Qi implique, au moins potentiellement, un risque pour ses propres intérêts, car si le monarque de ce pays ennemi obtient ses services cela lui vaudra un avantage militaire important, Pang Juan décide de convoquer Sun Bin, peut-être en lui offrant son appui ou encore en lui promettant un entretien avec le roi du Wei en vue d'une promotion. De plus, Pang Juan décide de garder secrète cette rencontre afin de ne pas éveiller les soupçons du Qi. Alerté par son départ, le souverain du Qi pourrait en effet le retenir sur son territoire afin que ses talents ne finissent pas aux mains de l'ennemi. Cependant, une fois Sun Bin arrivé au Wei, conscient de sa supériorité en matière de science militaire, Pang Juan, craignant que le nouveau venu lui fasse de l'ombre ou même le supplante à la tête des armées s'il parvient à s'entretenir avec le monarque, neutralise ce danger. Il obtient que lui soit infligé un châtiment corporel qui lui interdit désormais d'assurer une charge publique et même d'être reçu à la cour pour une audience. La manœuvre imaginée par Pang Juan vise ainsi à empêcher que le talent de Sun Bin soit mis au service d'un pays ennemi, tout en évitant que sa présence ne devienne un problème pour sa propre carrière. Par ailleurs, l'histoire de Sun Bin et Pang Juan obéit à une trame similaire à celle d'autres biographies d'acteurs de la vie politique des Royaumes Combattants. On peut citer par exemple la biographie de Zhang Yi où l'on retrouve une humiliation analogue: alors qu'il se trouve au service de la cour de Chu, Zhang Yi est accusé d'avoir volé un jade au monarque et subit, en conséquence, la peine de la bastonnade. C'est alors que son camarade Su Qin, conscient de la supériorité de Zhang Yi obtient pour ce dernier un poste d'officier dans le but de pouvoir l'utiliser ultérieurement, une fois acquises sa gratitude et sa confiance, dans une manœuvre diplomatique complexe dirigée contre le Qin ${ }^{49}$. Reflet fidèle

48. Shiji, 2162.

49. Shiji, LXX, 2279-2283. On retrouve certains éléments de cette trame dans la notice biographique de Fan Sui 范睢 qui subit un châtiment corporel ainsi que d'autres humiliations en raison d'une fausse accusation. Comme Sun Bin, il parvient à sauver sa vie en fuyant et en se réfugiant dans la clandestinité : Shiji, LXXIX, 2401-2402. En définitive, le complot de Pang Juan contre Sun Bin fait écho au complot du premier ministre de Qin, Li Si, contre son ancien condisciple Han Fei, qu'il fit condamner après l'avoir fait mander au Qin (Shiji, LXIII, 2155). Chez Sima Qian, le thème de l'humiliation, de la ruine de l'intégrité physique dans ses différentes variantes, parmi 
d'un contexte concurrentiel caractéristique de la vie politique des Royaumes Combattants regorgeant de machinations, d'intrigues et de trahisons, l'anecdote concernant Sun Bin montre l'esprit de ruse jouer sur la polarité du visible et de l'invisible, de l'évidence et du secret. Ainsi, Pang Juan conspire non seulement pour empêcher toute rencontre, mais aussi pour que son rival soit mis définitivement hors jeu en le condamnant à une invisibilité définitive (wujian) par un traitement qui, paradoxalement, accentue sa visibilité. Le châtiment corporel frappant Sun Bin implique une exclusion radicale. En Chine ancienne, les châtiments corporels comme le tatouage du visage ou, plus encore, l'amputation des pieds, entraînaient de terribles conséquences sociales. Les victimes de mutilations corporelles se voyaient répudiées et toute participation aux activités rituelles leur était désormais interdite. Évidemment, cette exclusion est d'autant plus forte que la difformité corporelle résulte d'une condamnation pénale ${ }^{50}$. La honte d'apparaître en public sous une apparence monstrueuse et effrayante provoquant une répulsion instinctive est plus lourde lorsqu'il s'agit d'une difformité porteuse d'un signe évident de criminalité et, de plus, irréversible. L'efficacité du châtiment (xing) réside, par conséquent, dans une transformation de la forme corporelle (xing*) telle que la corrélation entre la caractéristique morale de l'individu et ses traits physiques devient fixe et immuable. De ce point de vue, la manœuvre de Pang Juan contre Sun Bin opère sur ce rapport entre la dimension extérieure et intérieure de l'individu. Si bien que la personne mutilée se voit exposée et condamnée sous l'emprise du préjugé de la correspondance entre l'apparence extérieure et la réalité intérieure sans qu'il soit désormais nécessaire de déployer une quelconque habileté physiognomonique. La lecture de sa corporéité est à présent évidente. Le probable désir de Sun Bin d'apparaître et de se présenter devant le monarque pour faire reconnaître ses talents se voit frustré. En effet, le stratagème de Pang Juan condamne son rival non seulement à l'ostracisme, mais à désirer se réfugier dans l'anonymat et demeurer caché. Le châtiment

lesquelles la mutilation, représente un topos central et récurrent, celui du héros ridiculisé dont l'abjection physique finit par se transformer en signe d'élection et qui correspond, très probablement, à la tragédie vécue par Sima Qian lui-même. Comme on le verra plus loin, le châtiment corporel de Sun Bin le disqualifie complètement en tant que personnage public et le distingue en même temps comme individu exceptionnel. Dans le Shiji, du moins, loin de forger un destin tragique immuable et définitif, le châtiment corporel finit par ouvrir certaines alternatives.

50. Pour une étude plus complète des conséquences de ces châtiments corporels dans la Chine ancienne, voir Turner (1999). 
corporel subi marque à jamais son destin, comme l'indique son propre nom ${ }^{51}$, et en fait un être qui doit rester toujours caché, invisible (ce qui, d'une certaine manière, finit par jouer un rôle important dans son succès futur en rendant possible sa revanche). Ainsi se voit-il contraint de s'entretenir avec l'émissaire du Qi dûment protégé et à l'abri des regards. Après avoir convaincu cet envoyé spécial, il est à nouveau transporté clandestinement, caché dans une voiture couverte. Comme l'indique la suite de l'anecdote, sa réputation sera rétablie et il gravira l'échelle sociale jusqu'à devenir le conseiller du roi de Qi. Cependant, son aspect physique lui imposera, une fois de plus, non seulement de refuser la charge de commandant qui lui est offerte, au prétexte que cela n'est pas admissible pour un mutilé mais aussi de rester à l'intérieur d'un chariot, à l'abri, en position discrète, pour décider du plan de bataille et de la stratégie des armées $^{52}$.

\section{Manipulation de signes et leurres}

Cependant, si Sun Bin parvient à échapper à la fatalité de ce destin, c'est grâce à une action qui implique le dévoilement de l'intériorité grâce à la lecture de son apparence. En effet, après une parenthèse, le récit des péripéties de Sun Bin dans le Qi se poursuit ainsi :

[Tian] Ji aimait miser au jeu et il pariait souvent avec d'autres nobles aux courses de quadriges. Sunzi [Bin] observa les sabots de ses chevaux et s'aperçut que, du point de vue de leur qualité, ils n'étaient pas très éloignés de ceux du monarque. Il classa les chevaux en lice en excellents, moyens et inférieurs. Ainsi, Sunzi [Bin] dit à Tian Ji : «Pariez contre les chevaux du prince et je vous ferai gagner.» Tian Ji fit confiance à son choix et paria mille pièces d'or avec le monarque et les nobles. Après avoir inspecté la qualité [des chevaux], Sun Bin lui dit: «Opposez le groupe de vos chevaux inférieurs au groupe des meilleurs de votre adversaire, puis le groupe de vos meilleurs chevaux au groupe de ses moyens et, enfin, le groupe de vos chevaux

51. L'importance de cette marque corporelle trouve en effet un écho dans sa propre identité, dans le nom sous lequel il est connu. Le terme bin 臏 désigne l'os principal du genou et, par extension, la mutilation qu'il subit sous l'effet du couteau. De fait, il est fréquent à l'époque d'utiliser des noms, souvent attribués de manière posthume, qui peuvent être interprétés comme des épithètes exprimant un jugement sur les qualités d'un individu. Sur cette question, voir les contributions de Zhou Fagao (1958); Petersen (1992); et Goldin (2000).

52. Shiji, 2163. 
moyens au groupe de ses chevaux inférieurs.» À l'issue de la compétition des trois groupes, Tian Ji n'avait perdu qu'une seule course et avait remporté les autres, ce qui lui permit d'empocher les mille pièces d'or du roi. Pour le récompenser, Tian Ji introduisit Sunzi [Bin] auprès du roi du Wei. Le roi du Wei l'interrogea au sujet de la science militaire, après quoi il décida de le nommer conseiller ${ }^{53}$.

L'interprétation proposée de ce passage s'éloigne sur certains points de l'exégèse conventionnelle. Dans la phrase que j'ai décidé de traduire par «il observa les sabots de ses chevaux» (jian qi ma zu), les commentateurs traditionnels expliquent que le terme $z u$, qui signifie «pied» ou «sabot», se réfère, en l'occurrence, à la capacité des chevaux ${ }^{54}$; quant à l'expression que j'ai choisi de rendre par «après avoir inspecté la qualité [des chevaux]» (ji lin zhi), elle renverrait, toujours selon l'exégèse traditionnelle, au moment précédant immédiatement le départ de la course ${ }^{55}$. De telles affirmations ne sont pas convaincantes. La lecture de la première phrase retenue par ces commentaires semble forcée, alors que, au sens littéral, l'expression ne présente aucune difficulté et n'exige aucune explication. Il est évident que pour pouvoir déterminer la capacité et la force des chevaux, il est nécessaire de prêter attention à leurs sabots, partie essentielle de leur anatomie ${ }^{56}$. Pour ce qui est de la seconde phrase, je suis également partisan de son sens le plus littéral qui a trait à l'observation de la substance ou de la qualité interne des chevaux. De fait, dans un passage du Fengsu tongyi cette même expression verbale (ji lin) renvoie à l'examen minutieux d'une chose, et ne désigne pas la séquence temporelle précédant cet examen ${ }^{57}$.

Certes, Sun Bin met au point une combinaison astucieuse pour faire concourir les chevaux et c'est surtout cet art de classer les animaux puis de réorganiser la course en fonction d'un calcul logique qui lui permet de faire remporter la victoire. Mais il ne faut pas oublier que le succès de ce calcul dépend en premier lieu de sa capacité à déchiffrer correctement les traits physiques des chevaux et de pronostiquer par la lecture de signes extérieurs leurs différentes qualités de force et de résistance. Le fait que l'ascension de

53. Shiji, pp. 2162-2163.

54. Telle paraît être aussi la leçon suivie dans la version anglaise éditée par Nienhauser où ce passage est traduit ainsi: "[Sun Bin] noticed that the horse's speed was not much different»: (1994): 39.

55. Voir Zhang Dake (1990): 1337, n. 3 ; et Takikawa Kametarō ([1934] 1985) : 3304.

56. Dans le cas présent, Zhang Dake (1990) : 1336, n. 14, lit le passage comme je le fais et interprète le terme $z u$ comme faisant référence à la force des sabots des chevaux (馬的 腳力).

57. Fengsu tongyi xiaozhu 風俗通義校注, II, 128 . 
Sun Bin soit due, au moins au départ, non pas tant à un haut fait militaire ou à la démonstration d'une connaissance liée à des qualités stratégiques, qu'à une prédiction, est, sans doute, significatif. Le recouvrement de son honneur et la reconnaissance de ses qualités exceptionnelles dans l'art de la guerre sont dus, en réalité, à un geste qui l'apparente à celui d'autres célèbres physiognomonistes équins, comme, par exemple Bo Le ou Chen Pei, déjà mentionnés. En faisant référence à l'habileté de Sun Bin dans le maniement des techniques physiognomoniques permettant de prédire les qualités des êtres, cette partie du récit démontre la pertinence de l'observation pour "inspecter la substance interne" (lin zhi) et assurer le succès des calculs. Elle confirme aussi, bien que de manière implicite, le lien entre la prévision des événements et les aptitudes dans le domaine de la stratégie. Cette dernière association deviendra encore plus évidente au cours du récit.

Les années passent et la carrière militaire de Sun Bin connaît de notables succès, comme la victoire sur les armées du Wei lors de la célèbre bataille de Guiling ${ }^{58}$. Alors que Sun Bin dirige les opérations militaires depuis l'intérieur d'un char, le général Tian Jin inflige, en 353 av. J.-C., une sévère défaite aux troupes du Wei dans la localité de Guiling. Rappelons le contexte: un an auparavant, les dirigeants du Wei avaient lancé une opération militaire contre le Zhao en attaquant sa capitale, Handan. Lorsque, après des mois de combats épuisants, la ville assiégée par les meilleures troupes du Wei est sur le point de tomber, les dirigeants du Qi estiment que le moment est venu de fournir l'appui que ne cesse de leur réclamer le Zhao depuis longtemps ${ }^{59}$. Si l'on en croit le récit du Shiji, l'option initiale du général de Qi, Tian Ji, consistait à marcher sur Handan pour éviter que la ville ne tombât aux mains du Wei. C'est alors que Sun Bin avance l'idée d'un plan plus avantageux : profitant du fait que le gros des troupes du Wei se trouve à Handan, il propose de lancer une offensive contre la capitale du Wei, Daliang, car ainsi les troupes du Wei commandées par Pang Juan se verront dans l'obligation d'abandonner le siège et de se déplacer dans l'urgence pour venir à la rescousse de leur capitale. En une seule

58. Shiji, 2163. Pour une description complète de cette bataille, qui inclut les prémices, l'analyse tactique et les conséquences géopolitiques de l'événement, je suis les contributions de Sawyer (1999): 31-41. La renommée de cet affrontement armé acquise grâce à l'habileté stratégique de Sun Bin est illustrée par une sorte de proverbe très populaire, «Assiéger Wei pour sauver Zhao» (wei Wei jiu Zhao 圍魏救趙), rapporté et cité dans plusieurs sources écrites postérieures.

59. Bien que les noms des acteurs ne coïncident pas avec le récit du Shiji, le Zhanguo ce contient un passage exposant le débat présumé à la cour du Qi entre les partisans de l'intervention pour secourir le Zhao et les détracteurs d'une telle opération qui sont partisans de laisser le Wei conquérir le Zhao. Voir Zhanguo ce jian zheng, 504-505. 
manœuvre, le Zhao peut briser le siège tandis que le Qi obtient un avantage décisif pour affronter les soldats du Wei épuisés tant par le combat prolongé d'une année entière que par la fatigue causée par la précipitation du retour vers leur capitale. Tian Ji décide de suivre le conseil tactique de Sun Bin et parvient à écraser les troupes du Wei lors de cette mémorable bataille de Guiling .

Cependant, parmi les manuscrits exhumés en 1972 sur le site de Yinqueshan, dont on dit qu'ils feraient partie de l'œuvre perdue attribuée à Sun Bin, se trouve une série de lamelles de bambou intitulée «La capture de Pang Juan» (Qin Pang Juan). Ce texte peut fournir quelques informations supplémentaires à propos de la manœuvre utilisée par Sun Bin pour remporter la victoire au cours de cette bataille ${ }^{60}$. Si ce que rapporte cette version est fiable, le stratagème imaginé par Sun Bin serait quelque peu plus complexe et sophistiqué que la version conservée par le Shiji ${ }^{61}$. Face au siège de Handan par le Wei, Sun Bin aurait ordonné de sacrifier un certain nombre d'unités de sa propre armée en les envoyant à l'assaut de la ville de Pingling, située au sud-est du territoire du Wei et dotée d'un solide dispositif de défense. Comme prévu, les unités du Qi sont repoussées avec facilité par les régiments de cette cité. Puis Sun Bin fait mine de contre-attaquer de manière précipitée et prévisible: il ordonne une nouvelle fois que quelques unités légères se préparent à attaquer Daliang, la capitale du Wei, afin de provoquer la colère de Pang Juan et, en divisant ses effectifs, lui faire croire que ses troupes sont peu nombreuses ${ }^{62}$. Persuadé qu'il s'agit là d'une manœuvre douteuse et désespérée, confiant dans la supériorité de ses propres troupes, rassuré par la récente défaite du Qi à Pingling, et désireux, sans doute, de revendiquer sa victoire auprès de son souverain, Pang Juan ordonne qu'une grande partie de ses troupes regagne immédiatement la capitale pour écraser l'ennemi. Sun Bin anticipe le trajet du retour choisi par Pang Juan et y déploie le plus gros de ses troupes, jusque-là en réserve quelque part entre le Qi et le Wei. Bien reposées et dûment postées, elles attendent à Guiling, terrain propice et déjà reconnu, l'arrivée des troupes de Pang Juan pour les affronter avantageusement et les vaincre.

60. Voir Sun Bin bingfa jiaoli 孫臏兵法校理, 1-15.

61. La manœuvre consistant à neutraliser l'offensive lancée par un pays contre la capitale d'un autre grâce à une attaque menée contre le territoire du premier par un troisième pays qui se porte à la rescousse du deuxième pays est déjà mentionnée à plusieurs reprises dans le Zuozhuan. Selon cette source écrite, en 623 av. J.-C., les troupes du Chu assiègent la capitale du pays de Jiang. Une armée du Jin pénètre alors dans la capitale du Chu afin de libérer ainsi le Jiang (Chunqiu Zuozhuan zhu, 531). Il est probable que Pang Juan ait pensé, grâce aux faux indices fabriqués par Sun Bin, que les armées du Qi prévoyaient de réitérer cette vieille recette stratégique éculée.

62. Sun Bin bingfa jiaoli, 2 . 
En 341 av. J.-C., plus d'une décennie après ce premier succès militaire sur le Wei, l'occasion se présente enfin à Sun Bin de se venger définitivement de Pang Juan. Mais selon le récit tiré des matériaux provenant de Yinqueshan, ce dernier aurait pu réchapper de la bataille de Guiling. La dernière partie de l'anecdote du Shiji offre au lecteur le récit suivant de la bataille de Maling:

Treize ans plus tard, les pays de Wei et de Zhao lancèrent une offensive conjointe contre le Han. Le Han informa le Qi de sa situation désespérée et le gouvernement de Qi décida d'envoyer ses troupes, sous le commandement de Tian Jin, directement contre Daliang. Lorsque le général Pang Juan apprit cela, il leva immédiatement le siège de Han et décida de retourner à Wei. Les armées du Qi poursuivirent leur route et se dirigèrent vers l'Ouest. Sun Bin dit à Tian Ji: «Les soldats des trois pays issus du démembrement du Jin [le Han, le Wei et le Zhao] sont simples, impétueux et courageux, et ils méprisent le potentiel du Qi car, chez eux, notre pays est considéré comme peuplé de lâches. Qui est compétent dans les arts militaires s'adapte au potentiel stratégique de l'adversaire et l'utilise à son profit. Selon la science de la guerre, qui parcourt mille lieues en quête d'un avantage subira la chute de ses chefs et qui parcourt cinq cent lieues à la recherche d'un avantage atteindra son objectif avec seulement la moitié de ses effectifs ${ }^{63}$. Je vous prie d'ordonner à nos troupes qu'en arrivant aux frontières de Wei, elles allument cent mille torches, le jour suivant, seulement cinquante mille et le troisième jour, enfin, seulement trente mille.»

Au bout de trois journées de marche, Pang Jan exulte et s'exclame : «Il m'apparaît avec certitude que les armées du Qi sont faites de lâches. Elles sont sur notre territoire depuis seulement trois jours et le nombre des pertes en officiers et soldats dépasse déjà la moitié de leurs effectifs.» En conséquence, il laissa le gros de son infanterie et, accompagné de quelques unités d'élite, il se lança à la poursuite des troupes de Qi, parcourant en une seule journée la distance que l'on franchit normalement en deux. Sun Bin fit une estimation des distances et conclut qu'il arriverait dans la localité de Maling à la tombée de la nuit. La route conduisant à Maling était encaissée, avec de nombreux obstacles et défilés où pouvoir poster des soldats dissimulés, si bien que Sun Bin fit une large incision sur un grand arbre qui se trouvait là et écrivit sur le blanc de son écorce: «Pang Juan est mort au pied de ce grand arbre.» Puis, il ordonna que dix mille arbalétriers parmi les plus habiles des régiments du Qi se postent secrètement sur la route de Maling avec la consigne suivante: «Au crépuscule, lorsque vous apercevrez un feu, tirez ensemble à

63. Il s'agit, très probablement, d'une citation tirée des écrits militaires traditionnellement attribués à son ancêtre Sun Wu, dans l'œuvre duquel se trouve un passage très proche: Shi yi jia zhu Sunzi jiao li, VII, 137-138. 
volonté.» À la tombée de la nuit, Pang Juan arriva à l'endroit où se trouvait le grand arbre qui avait été marqué et, après avoir aperçu l'inscription, alluma une torche. Il n'avait pas terminé de lire l'inscription que les dix mille arbalétriers décochèrent leurs flèches en même temps. Les soldats du Wei tombèrent victimes du chaos et du désordre. Sachant sa clairvoyance flétrie et ses armées bientôt battues, Pang Juan se trancha la gorge après s'être exclamé: «J'ai contribué à asseoir le renom de cet infâme ${ }^{64}$.» Pour de tels faits, le nom de Sun Bin fut connu dans le monde entier et sa science militaire fut transmise de génération en génération ${ }^{65}$.

Alliés à leur ancienne victime, le Zhao, les dirigeants du Wei poursuivent leur politique agressive d'expansion et décident d'attaquer cette fois-ci le pays de Han. Devant la demande d'aide de la part du Han et, à nouveau, après un débat à la cour recueilli dans le Zhanguo ce, le monarque du Qi décide d'intervenir et de s'opposer à l'offensive du Wei ${ }^{66}$. La stratégie dissuasive du Qi recourt, une fois de plus, à l'habituelle formule consistant à donner l'assaut à la capitale du Wei pour, ce faisant, obliger ses troupes à abandonner le siège de Han. Cependant, derrière cette manœuvre simple en apparence, Sun Bin prépare un piège plus retors pour tromper son adversaire. Au lieu de réaliser son attaque contre Daliang, les armées commandées par Tian Ji, mais sous la direction tactique de Sun Bin, feignent de se replier en direction du nord-ouest. En exécutant un tel mouvement, il vise à piquer l'orgueil de son ennemi et à exploiter à son profit le désir de ce dernier de réaliser un gain inespéré. Sachant que les armées du Qi ont la réputation d'être timorées aux yeux du Wei, Sun Bin fait croire que sa fuite est due à la nouvelle de l'arrivée imminente des troupes commandées par Pang Juan. Selon toute probabilité, la perspective d'une victoire facile face à un ennemi en fuite pousse le monarque du Wei, au lieu de garder ses garnisons dans la capitale, à les envoyer s'emparer des forces du Qi et à mettre à leur tête l'héritier sans expérience, le prince Shen. Afin d'inciter Pang Juan à la précipitation et, par conséquent, lui faire commettre une erreur, Sun Bin multiplie les fausses pistes qui renforcent son ennemi dans ses erreurs de jugement. Pang Juan tombe dans le piège tendu par Sun Bin parce qu'il se laisse guider par l'interprétation de ces indices falsifiés qui semblent corroborer sa perception de l'état moral des troupes du Qi. Si, comme on l'a signalé plus haut, la peine infligée à Sun Bin, comme conséquence de la manœuvre ourdie par Pang Juan à la cour du Wei, visait à son exclusion sociale en vertu de la

64. Selon un fragment du Zhanguo ce, le général Pang Juan aurait été capturé vivant lors de la bataille de Maling: Zhanguo ce jian zheng, XXIII, 1337.

65. Shiji, 2164-2165.

66. Zhanguo ce jian zheng, VIII, 508. 
force des préjugés concernant son stigmate d'amputé, la vengeance de Sun Bin qui s'ensuit s'organise selon une logique analogue. En effet, ces torches dont le nombre ne cesse de diminuer sont interprétées comme les signes probants des dispositions morales de l'adversaire. Au rebours de l'expertise dont fait preuve Sun Bin lors de l'examen des chevaux, la déduction que réalise Pang Juan à partir d'indices visibles, certainement aiguillonnée par son désir de revanche, est telle qu'il commet une erreur de prévision sur l'état des troupes du Qi. Ainsi, parce qu'il connaît la force de telles conventions et de tels préjugés, Sun Bin anticipe la réponse anticipée de Pang Juan et parvient de ce fait à le dominer. Ce jeu permanent sur les signes et les indices, les fausses et les vraies traces culmine avec l'inscription du message destiné à Pang Juan sur l'écorce d'un arbre. D'une certaine manière, ce geste condense la leçon qu'il convient de tirer de l'anecdote à propos des risques et des dangers de l'anticipation. En observant les torches, Pang Juan croit anticiper les mouvements de son rival alors qu'en réalité il ne fait qu'obéir au plan prévu par Sun Bin. La scène finale illustre la faillite de Pang Juan dans l'interprétation correcte tant des indices révélateurs que des fausses pistes. Aveugle jusque-là devant cette chaîne de leurres, lorsqu'il déchiffre l'inscription sur l'arbre il est déjà trop tard. Ce n'est qu'alors qu'il réussit à comprendre qu'il vient d'être victime d'un piège. Et qu'il parvient à prédire le brillant destin qui attend Sun Bin et qu'il a lui-même contribué à créer.

\section{BIBLIOGRAPHIE}

\section{Sources primaires}

Boshu Laozi jiao zhu 帛書老子校注. Édition établie par Gao Ming 高明. Beijing: Zhonghua shuju, 1996.

Chunqiu fanlu yizheng 春秋繁露義證. Édition établie par Zhong Zhedian 鐘哲點. Beijing: Zhonghua shuju, 1992.

Chunqiu Zuozhuan zhu 春秋左傳注. Édition établie par Yang Bojun 楊伯君. Beijing: Zhonghua shuju, 1995.

Da Dai Lijijiegu 大戴禮記解詁. Édition établie par Wang Pinzhen 王聘珍. Beijing: Zhonghua shuju, 1983.

Fengsu tongyi xiaozhu 風俗通義校注. Édition établie par Wang Liqi 王力器. Beijing: Zhonghua shuju, 1981.

Guanzi jiao zhu 管子校注.Édition établie par Li Xiangfeng 黎翔鳳. Beijing : Zhonghua shuju, 2000.

Guoyu 國語. Édition établie par Shanghai Shifan daxue guji zhengli xiaozu 上海師範 大學古籍整理小組. Shanghai: Shanghai guji chubanshe, 1990. 
Han Feizi xin jiaozhu 韓非子新校注. Édition établie par Chen Qiyou 陳奇猷. Shanghai: Shanghai guji chubanshe, 2000.

Hanshu 漢書. Par Ban Gu 班固. Beijing: Zhonghua shuju, 1956.

He Guanzi hui jiao jizhu 鶴冠子汇校集注. Édition établie par Huang Huaixin 黃怀信.

Beijing: Zhonghua shuju, 2004.

Huainanzi jiaoyi 淮南子校譯. Édition établie par Zhang Shuangdi 張雙棣. Beijing:

Beijing Daxue chubanshe, 1997.

Huangdi neijing zhangju suoyin 黃帝內經章句索引. Édition établie par Ren Yingqiu

任應秋. Beijing: Renmin weisheng chubanshe, 1986.

Kongcongzi 孔叢子. Édition établie par Wang Junlin 王鈞林 \& Zhou Haisheng 周海 生. Beijing: Zhonghua shuju, 2009.

Kongzi jiayu shu zheng 孔子家語疏証. Édition établie par Chen Shike 陳士珂. Shanghai: Shanghai shudian, 1980.

Liji jijie 禮記集解. Édition établie par Sun Xidan 孫希旦. Beijing: Zhonghua shuju, 1996.

Lunheng jiaoshi 論衡校釋. Édition établie par Huang Hui 黃暉. Beijing: Zhonghua shuju, 1990.

Lüshi chunqiu xin jiaoshi 呂氏春秋新校釋. Édition établie par Chen Qiyou 陳奇猷. Shanghai: Shanghai guji chubanshe, 2002.

Mozi jiangu 墨子間詁. Édition établie par Sun Yirang 孫詒讓. Taibei : Shijie, 1974.

Shang jun shu zhuizhi 商君書錐指. Édition établie par Jiang Lihong 蔣禮鴻. Beijing: Zhonghua shuju, 1986.

Shiji 史記. Par Sima Qian 司馬遷 (ca. 145-ca. 86 BCE). Beijing: Zhonghua shuju, 1959.

Shiyi jia zhu Sunzi jiaoli 十一家注孫子校理. Édition établie par Yang Bingan 楊丙安. Beijing: Zhonghua shuju, 1999.

Shuo yuan jiaozheng 說苑校証. Édition établie par Xiang Zonglu 向宗魯. Beijing: Zhonghua shuju, 1989.

Sun Bin bingfa jiaoli孫臏兵法校理. Édition établie par Zhang Yunze 張震澤. Beijing: Zhonghua shuju, 1984.

Tai gong Liu Tao jinzhu jinyi 太公六韜今註今譯. Édition établie par Xu Peigen 徐培 根. Taibei: Taiwan shangwu, 1984.

Wuzi jinzhu jinyi 吳子今註今譯. Édition établie par Fu Shaojie 傅紹傑. Taibei : Taiwan shangwu, 1985.

Yinqueshan Hanmu zhu jian 銀雀山漢墓竹簡. Édition établie par Yinqueshan Hanmu zhujian zhengli xiaozu 銀雀山漢墓竹簡整理小組. Beijing: Wenwu chubanshe, 1985.

Yi Zhoushu hui jiao ji zhu 逸周書彙校集注. Édition établie par Huang Huaixin 黃懷 信. Shanghai : Shanghai guji chubanshe, 2007.

Xinshu jiaozhu 新書校注. Par Jia Yi 賈誼. Beijing: Zhonghua shuju, 2000.

Xinxu jinzhu jinyi 新序今注今譯. Édition établie par Liu Xiang 劉向. Tianjin: Tianjin guji chubanshe, 1988.

Xunzi jijie 荀子集解. Édition établie par Wang Xianqian 王先謙. Beijing: Zhonghua shuju, 1997. 
Yantielun jiao zhu 鹽鐵論校注. Édition établie par Wang Liqi 王利器. Beijing: Zhonghua shuju, 1992.

Zhanguo ce jian zheng 戰國策戔證. Édition établie par Fan Xiangyong 范祥雍. Shanghai: Shanghai guji chubanshe, 2008.

Zhouli zhengyi 周禮正義. Édition établie par Sun Yirang 孫詒讓. Beijing: Zhonghua shuju, 2008.

Zhouyi zhengyi 周易正義. Édition établie par Liu Yujian 劉玉建. Jinan: Qi Lu shushe, 2005.

Zhuangzi jishi 莊子集釋. Édition établie par Guo Qingfan 郭慶藩. Beijing : Zhonghua shuju, 1968.

\section{Sources secondaires}

Brown Miranda \& Bergeton Uffe (2008). «Seeing Like a Sage : Three Takes on Identity and Perception in Early China». Journal of Chinese Philosophy, 35.4: 641-662.

Csiskszentminalyi Mark (2011). «Allotment and Death in Early China». In Amy Olberding \& Philip J. Ivanhoe (dir.), Mortality in Traditional Chinese Thought. Albany, State University of New York Press: 177-190.

Debru Armelle (2007). «Signes, indices et inférences en médecine antique». In Denis Thouard (dir.), L'Interprétation des indices. Enquête sur le paradigme indiciaire avec Carlo Ginzburg. Villeneuve d'Asq, Presses Universitaires du Septentrion: 175-188.

Galvany Albert (2011). «Philosophy, biography, and anecdote: on the portrait of Sun Wu ». Philosophy East \& West, 61.4: 630-646.

GinZburg Carlo (1989). «Traces. Racines d'un paradigme indiciaire». In Mythes, emblèmes, traces. Morphologie et histoire. Paris, Flammarion.

Goldin Paul R. (2000). «Personal Names in Early China: A Research Note». Journal of the American Oriental Society, 120.1: 77-81.

HARDY Grant (1999). Worlds of Bronze and Bamboo : Sima Qian's Conquest of History. New York, Columbia University Press.

Hu Pingsheng 胡平生 (1998). «Fuyang Shuanggudui Han jian shushu shu jian lun 阜陽雙古堆漢簡數術書簡論》. Chutu wenxian yanjiu 出土文獻研究 (1998.4): $12-30$.

HulsewÉ A. F. P. (1979). «Watching the vapours: an ancient Chinese technique of prognostication». Nachrichten, 125: 40-49.

KALINOWSKI Marc (1999). «La rhétorique oraculaire dans les chroniques anciennes de la Chine. Une étude des discours prédictifs dans le Zuozhuan». Extrême-Orient Extrême-Occident, $21: 37-65$.

Levi Jean (1989). Les Fonctionnaires divins. Politique, despotisme et mystique en Chine ancienne. Paris, Éditions du Seuil.

Levi Jean (1995). La Chine Romanesque. Paris, Éditions du Seuil.

Li Ling 李零 (1993). Zhongguo fangshu gaiguan 中國方術概觀, Beijing, Renmin chubanshe.

LI Wai-yee (2007). The Readability of the Past in Early Chinese Historiography. Cambridge, Harvard University Press. 


\section{Albert Galvany}

MA Mingda 馬明達 (1982). «Juyan Han jian 'Xiang jiandao'ce chutan 居延漢簡相劍 刀冊初探》. Dunghuangxue jikan 敦煌學輯刊 (1982.3): 79-89.

Manetti Giovanni (1993). Theories of the Sign in Classical Antiquity, Bloomington, Indiana University Press.

NienHauser William H.(ed.) (1994). The Grand Scribe's Records, vol. VII. Bloomington, Indiana University Press.

Nylan Michael \& Csikszentminalyi Mark (2003). "Constructing Lineages and Inventing Traditions through Exemplary Figures in Early China». T'oung Pao, 89: 59-99.

Pankenier David (2013). Astrology and Cosmology in Early China. Conforming Earth to Heaven. Cambridge, Cambridge University Press.

Petersen Jens O. (1992). «What's in a name? On the Sources concerning Sun Wu». Asia Major, $5.1: 1-31$.

PINES Yuri (2002). Foundations of Confucian Thought. Intellectual Life in the Chunqiu Period, 722-453 B.C.E. Honolulu, University of Hawai'i Press.

SAwYer Ralph D. (1999). Sun Pin. Military Methods of the Art of War. New York, Barnes \& Nobles.

Sмiтн Kidder (2003). «Sima Tan and the Invention of Legalism, Daoism et cetera». The Journal of Asian Studies, 62.1 : 129-156.

TAKIKAWA Kametarō 瀧川龜太郎 ([1934] 1985). Shiki kaichu kōshō 史記會注考證. Taibei, Hongshi chuban.

Turner Karen (1999). «The Criminal Body and the Body Politic: Punishments in Early Imperial China». Cultural Dynamics, 11.2: 237-254.

VAndermeersch Léon (2013). Les Deux Raisons de la pensée chinoise. Divination et idéographie. Paris, Gallimard.

ZHANG Dake 張大可 (1990). Shiji quanti xinzhu 史記全體新注. Xi'an, Sanqin chubanshe.

ZHANG Duansui 張端穗 (1987). Zuozhuan sixiang tan wei 左傳思想探微. Taibei, Xuehai.

ZHONG Shaoyi 鍾少異 (1994). «Gu xiangjian shu chulun 古相檢術初論》. Kaogu 考 古 319:358-362.

Zhou Fagao 周法高 (1958). Zhou Qin mingzi jiegu huishi 周秦名字解詁彙. Taibei, Zhonghua congshu.

ZHu Pingyi 祝平一 (1990). Handai de xiangrenshu 漢代的相人術. Taibei, Xuesheng shuju. 


\section{GLOSSAIRE}

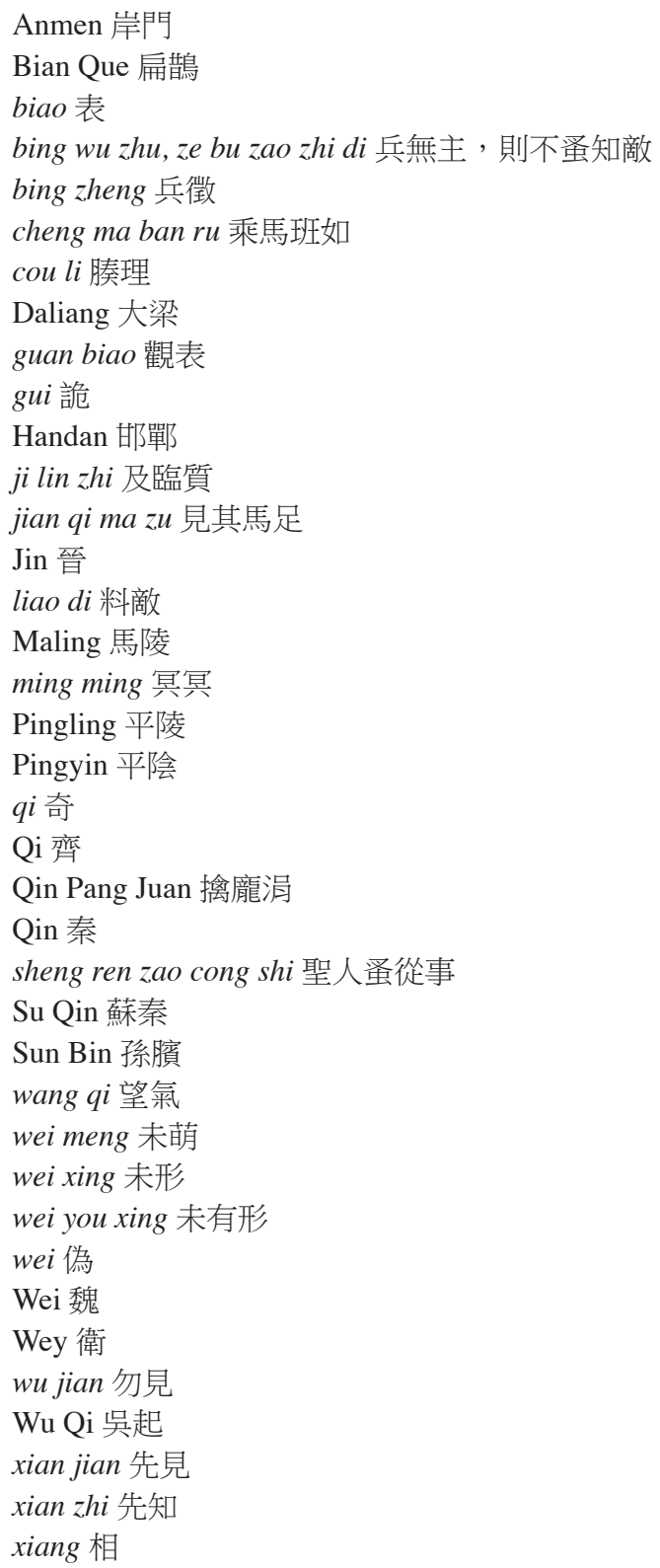


Albert Galvany

Xihe 西河

xing fa 形法

xing 刑

xing*形

Yinqueshan 銀雀山

yu jian 豫見

$z h a$ 詐

zheng 徵

$z u$ 足 